\title{
Erratum
}

17. Poleshuck EL, Giles DE, Tu X (2006) Pain and depressive symptoms among financially disadvantaged women's health patients. J Womens Health 15:182-193

18. Price DD (2000) Psychological and neural mechanisms of the affective dimension of pain. Science 288:1769-1772

19. Spitzer M (2016) Armut tut weh. Nervenheilkunde 35:185-18920

20. Randles D, Heine SJ, Santos N (2013) The common pain of surrealism and death: Acetaminophen reduces compensatory affirmation following meaning threats. Psychol Sci 24:966-973
Schmerz 2018 · 32:151

https://doi.org/10.1007/s00482-018-0285-7

Online publiziert: 29. März 2018

(c) Deutsche Schmerzgesellschaft e.V. Published by Springer Medizin Verlag GmbH, ein Teil von Springer Nature - all rights reserved 2017, korrigierte Publikation März 2018

CrossMark

D. Feierabend · J. Walter $\cdot$ R. Kalff · R. Reichart

Klinik und Poliklinik für Neurochirurgie, Universitätsklinikum Jena, Jena, Deutschland

\section{Erratum zu: Armut und Schmerz}

\section{Erratum zu:}

\section{Schmerz 2017}

https://doi.org/10.1007/s00482-017-

0259-1

Liebe Leserinnen und Leser,

bitte beachten Sie, dass die zunächst online veröffentliche Version dieses Artikels korrigiert wurde. Die ehemalige Tab. 1 ist entfallen und die Angaben $\mathrm{zu}$ den ethischen Richtlinien wurden angepasst.

Der Originalartikel wurde korrigiert.

\section{Korrespondenzadresse}

\section{Dr. D. Feierabend}

Klinik und Poliklinik für Neurochirurgie,

Universitätsklinikum Jena

Am Klinikum 1, 07747 Jena, Deutschland

Denise.Feierabend@med.uni-jena.de 